\title{
II Ruolo dei Tassi di Cambio nella Crescita dell'Economia Albanese
}

\author{
Prof. Ass. Alqi Naqellari \\ Lecturer in Mediterranean University in Tirana and in U."Ismail Qemali" Vlora, Albania \\ alqinaqellari@gmail.com \\ MA: Sonila Nikola \\ Lecturer in Mediterranean University in Tirana \\ soni_nikolla@yahoo.it \\ Dr. Altin Hoti \\ Dean. University "Luarasi” Tirana, Albania \\ altin.hoti@yahoo.com
}

Doi:10.5901/ajis.2013.v2n3p109

\section{Abstract}

The banking sector in Albania since the 90s had the aim of adapting the change of the economic relations of private property. It was decided to adapt a two-tiered system, similar to that of Western Europe, the Central Bank as the first level bank and commercial banks considered as level second banks. In this paper we will address the role of exchange rates in the rate of economic policy support the growth of economy. The conclusion of the material in concrete terms is that the exchange rate should be fixed and not flexible, because the role of the Central Bank shall be limited to the money market as a result of the free circulation of the euro and the dollar. Public expenditure in terms of realization of flexible exchange rates have no impact on economic growth. In this way, with flexible exchange rates, the limited impact of the Central Bank and the lack of influence of government have meant that the role of economic policy in the improvement of macroeconomic indicators is very small. They have little impact on GDP growth, reduce unemployment rate and inflation stability. These indicators, in the condition in which it is located, the Albanian economy, are evaluated when the currency is concentrates in the Central Bank and when applying fixed exchange rates.

Keywords: Banca Centrale, Banca commerciale, tassi di cambio fissi e variabili, la politica monetaria e fiscale, i prestiti, i depositi, libera circolazione, ecc.

\section{Introduzione}

Dopo gli anni '90, l'economia albanese ha iniziato a fare cambiamenti significativi cominciando a trasformarsi in un' economia di mercato. Le modifiche iniziarono nel campo della legislazione, l'adozione della legislazione con quello dell'Occidente. A sostegno il processo di privatizzazione della proprietà è iniziato bene. A questo proposito sono stati sviluppati una serie di programmi per la privatizzazione degli abitazioni, delle imprese di servizi e delle imprese del commercio, dell'industria leggera e alimentare, della rete telefonica fissa e mobile dino ad arrivare alla privatizzazione dei settori strategici. A sostegno di questa nuova struttura di proprietà sono stati sviluppati una serie di programmi per i cambiamenti funzionali e organizzativi nel settore bancario. E 'stata privatizzata l'ex Banca del Risparmio, interamente controllata dallo stato, ed è stato permesso l'apertura di banche commerciali private. II tasso di cambio era flessibile ed è stato permesso la libera circolazione dell'euro e del dollaro. Le banche avevano il diritto di accettare depositi e fornire prestiti in euro e dollaro. Sono queste le ragioni e le loro conseguenze sugli indicatori macroeconomici che ci hanno spinto a fare un' analisi sul ruolo del tasso di cambio flessibile sulla crescita economica nelle attuali condizioni della nostra economia.

\subsection{Il contenuto del materiale}

1. L'analisi del modello 
Il ruolo del tasso di cambio flessibile sulla crescita economica in una piccola economia aperta

2. Il ruolo del tasso di cambio fisso sulla crescita economica in una piccola economia aperta

\section{L'analisi del modello.}

\subsection{La curva IS}

In questa analisi verrà utilizzato il modello di Mundell-Fleming per le piccole economie aperte come l'economia albanese per un periodo di breve termine. La curva IS non la considereremo come curva di equilibrio che collega il reddito con il tasso di interesse. Utilizzeremo il tasso di cambio invece del tasso di interesse esprimendo la relazione in equilibrio tasso di cambio - reddito. Considerando il tasso di cambio flessibile come lo è oggi in Albania. II tasso di cambio flessibile è il tasso di cambio tra la valuta nazionale e le valute estere il quale viene determinato dalla domanda e dall'offerta nel mercato monetario. In questo modo il tasso di cambio non è determinato dalla Banca Centrale ma è determinato nel mercato. La Banca Centrale interviene solo in casi estremi, quando ci sono degli eccessi da parte delle entità economiche sul mercato.

Il rapporto tra i prezzi interni con quelli stranieri è fisso visto che in generale l'inflazione è importato.

\subsection{La costruzione della curva NX (le esportazioni nette).}

Partendo dai fati della tabella n.1 costruiamo la curva delle esportazioni nette rispetto ai tassi di cambio. Per la sua costruzione si prende il lato sinistro degli assi delle coordinate visto che le esportazioni nette sono di negative. Nel asse ascisse $\mathrm{X}$ ci sono le esportazioni nette, mentre nell'asse delle ordinate $Y$ abbiamo messo il tasso di cambio del lek per un euro, il che significa che, come ci si allontana dall'origine la valuta locale si deprezza di più. Per il periodo 2009-2012, questa curva è inclinata positivamente il che significa che maggiore è il deprezzamento della moneta locale vengono migliorati le esportazioni nette. La curva NX (le esportazioni nette) la utilizziamo per costruire la curva dei risparmi investimenti oppure la curva IS.

Tabella 1: La relazione tra il tasso di cambio e le esportazioni nette

\begin{tabular}{|c|c|c|c|c|c|}
\hline \multirow[t]{2}{*}{ Anni } & \multirow{2}{*}{$\begin{array}{l}\text { Tasso di } \\
\text { cambio }\end{array}$} & \multirow{2}{*}{$\begin{array}{c}\text { Bilancio } \\
\text { Commerciale (-) }\end{array}$} & Esportazioni & Importazioni & \multirow{2}{*}{$\begin{array}{l}\text { Bilancio commerciale } \\
\text { In ml/lekë }\end{array}$} \\
\hline & & & Milioni di EUR & & \\
\hline 2004 & 126.3 & 1276 & 485 & 1762 & 161,158 \\
\hline 2005 & 122.6 & 1476 & 530 & 2006 & 180,958 \\
\hline 2006 & 123.8 & 1659 & 630 & 2289 & 205,384 \\
\hline 2007 & 121.8 & 2104 & 786 & 2890 & 256,267 \\
\hline 2008 & 123.8 & 2431 & 917 & 3348 & 300,958 \\
\hline 2009 & 137.9 & 2304 & 750 & 3054 & 317,722 \\
\hline 2010 & 138.8 & 2082 & 1171 & 3254 & 288,982 \\
\hline 2011 & 138.9 & 2241 & 1405 & 3647 & 311,275 \\
\hline 2012 & 139.6 & 1999 & 1525 & 3524 & 279,060 \\
\hline
\end{tabular}

Fonte: Banca dell'Albania. Rapporto Statistico, Dicembre 12(1) 2012. P.5. Rapporto Statistico Mensile, 02/2013.

Fig.1: Curva delle esportazioni nette

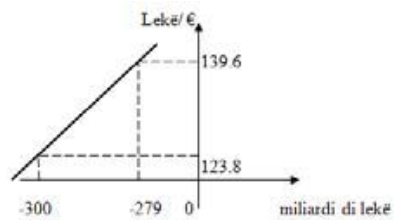

Dai dati della tabella e dal grafico, è chiaro che la curva delle esportazioni nette rispetto a tassi di cambio è inclinata 
positivamente. Non è simile al caso teorico quando relativamente al reddito consideriamo il tasso di interesse i . Nel caso di utilizzo dei tassi di cambio, per quanto viene apprezzato l'euro le esportazioni nette crescono di più avvicinandosi al zero.

II tasso di cambio lek/euro per gli anni 2007-2013 è stato in aumento, avendo cosi il deprezzamento del lek in contro l'apprezzamento dell'euro. Questo ha reso che le esportazioni crescano più rapidamente delle importazioni ed il rapporto esportazione / importazione nel 2012 è 1/2.3 quando nel 2007 era 1/3.67. II deprezzamento della valuta locale è associata ad un aumento delle esportazioni nette. Nel saldo totale del commercio dal -2431 milioni di euro nel 2008 è a 1.999 milioni di euro nel 2012. Questi rapporti portano la curva per le esportazioni nette (NX) ad una pendenza positiva.

Se si considera la forza di connessione tra i tassi di cambio con gli indicatori di cui sopra per il periodo 2009-2012 dovremmo avere $0,85,0,96$ e 0,78. Ci sono dei legami più stretti tra le esportazioni ed il tasso di cambio. Questa connessione è circa normale visto che il deprezzamento del lek viene accompagnato con l'aumento dei prezzi delle merci importate e quindi con la loro riduzione e l'aumentare delle esportazioni dei beni nazionali che diventano a sua volta più desiderabili. Le cifre nel saldo commerciale sono negative. In questo modo diminuisce la differenza tra le esportazioni e le importazioni.

\subsection{La costruzione della curva IS (investimenti-risparmi).}

Prendendo di riferimento la costruzione della curva NX e la sua connessione con il semplice modello Keynesiano costruiamo la curva IS. Nel quadrato a) abbiamo messo la curva NX. Nel quadrato b) abbiamo messo il modello semplice Keynesiano dell'equilibrio dei costi. Nel quadrato d) abbiamo messo la curva delle esportazioni nette e nell'quadrato c) abbiamo costruito la curva IS. Prendendo i dati dalla tabella 1 e 3 creiamo questo relazione:

Tab. 2: La relazione tra PIL, NX e i tassi di cambio per il periodo 2008-2012

\begin{tabular}{|c|c|c|c|}
\hline Anni & $\begin{array}{c}\text { PIL } \\
\text { Milioni di lekë }\end{array}$ & $\begin{array}{c}\text { NX } \\
\text { Milioni lekë }\end{array}$ & $\begin{array}{c}\text { Tasso di cambio } \\
\text { Lekë/euro }\end{array}$ \\
\hline 2008 & $1,089,293$ & -300 & 123.8 \\
\hline 2012 & $1,307,899$ & -279 & 139.6 \\
\hline
\end{tabular}

Utilizzando i dati degli anni 2008 e 2012 la curva IS viene è quella della Fig.2.

La curva IS sarà di pendenza più in alto a causa dell'aumento del tasso di cambio dell'euro, oppure il deprezzamento del lek porterà ad un aumento delle esportazioni nette tramite la riduzione delle importazioni e l'aumento delle esportazioni. L'aumento delle esportazioni nette sposta in alto la curva del costo del modello semplice Keynesiano portando all'aumento dei ricavi. In questo modo la curva IS accompagnerà la tendenza della curva NX.

Fig.2: La derivazione della curva IS

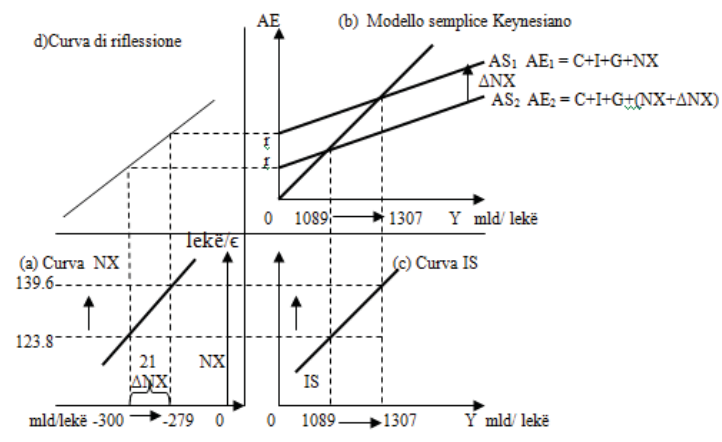

Se mettiamo a confronto questo modello con il modello originale $M-F$, dove avevamo la relazione tra la i e la y, allora noteremo che nelle condizioni dell'economia albanese non abbiamo la stessa posizione delle curve. In una situazione normale la curva IS avrà pendenza negativa. Perché è successo questo fenomeno continueremo a spiegarlo utilizzando i dati degli ultimi anni. 


\subsection{La Curva LM.}

Le suddette condizioni vengono utilizzate nella costruzione della curva LM. Ulterioriormente si aggiunge che l'offerta di moneta $\mathrm{M}$ è una variabile esogena che viene controllato dalla Banca Centrale. II tasso di interesse interno viene considerato senza influenza. Questo perché il tasso di interesse di base della moneta lek (REPO) non ha seguito il tasso di interesse di base dell'euro e del dollaro. Il cambio dell'euro di oggi è dello $0,5 \%$, mentre quella del lek è del $3,75 \%$. Non associare il tasso dell'euro o del dollaro dal tasso del lek ha portato a non correre della valuta in Albania sotto forma di investimenti in titoli o in depositi. In titoli di Stato Albanesi le banche estere operanti in Albania investono la maggior parte dei loro depositi in valuta nazionale. Solo il $40 \%$ dei crediti viene dato il lek perché il $60 \%$ di loro sono in valuta estera, così il resto va al acquisto dei titoli di Stato. Dal estero non abbiamo afflussi di valuta destinata allo scambio e destinata inoltre per tali attività. In questo modo il tasso di interesse del lek non ha alcun collegamento diretto al tasso di interesse estero. La diminuzione oppure la sua crescita non ha alcuna influenza sul flusso in entrata 0 in uscita. È per questo motivo che noi non lo consideriamo sulla pendenza della curva LM. Vale la pena di notare che il declino del tasso di interesse di base del lek ha poca o nessuna influenza sulla crescita o sulla riduzione dei tassi di interesse su depositi e dei prestiti in valuta estera. Questo perché il Lek è stato utilizzato dalle banche estere in base ai loro interessi. Per loro l'euro ed il dollaro sono le monete di riferimento indipendentemente dalle politiche applicate dalla Banca Centrale dell'Albania. Per loro non vi è alcun problema se ci sono delle modifiche del tasso base da parte della Banca Centrale. Per le banche straniere è importante l'azione della Banca centrale europea sull'euro. E 'la moneta più adatta nel settore del credito ed è alla pari al lek per quanto riguarda i depositi.

Per la curva $L M$ esistono due equazioni: $M / P=L(i, Y)$ e $L=k Y$ - hi.

II primo mostra l'equilibrio nel mercato monetario ed il secondo gli elementi dai quali dipende la domanda di moneta. La domanda di moneta è negativamente correlata con $\mathbf{i}$ ed $\mathbf{h}$, e correlata positivamente con $\mathbf{k}$ e $\mathbf{Y}$. II tasso di interesse i non ha alcun effetto sulla domanda di moneta, quindi non lo prendiamo in considerazione. I motivi per cui non viene considerato li abbiamo spiegato sopra. Così possiamo scrivere $L=k Y$ e dopo in equilibrio avremo :

$M / P=k Y M=k^{*} P Y M=k$ * PIL $k=M / P I L$, il che significa la quantità di moneta per 1 lek del valore dei prodotti.

Queste equazioni ci danno una curva LM verticale come mostrato in Fig. 3. La curva è verticale perché il tasso di cambio non può essere inserito nell'equazione LM. Con un tasso di interesse interno dato, l'equazione LM determina il reddito complessivo, indipendentemente dal tasso di cambio.

Fig. 3: La derivazione della curva LM

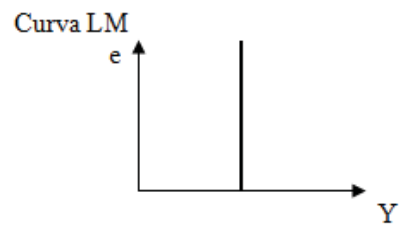

Di seguito sono riportati alcuni dati relativi agli indicatori monetari dell'economia albanese rispetto al PIL 2008-2011. Per il 2012 non ci sono dati sul PIL, ma secondo INSTAT rallenta rispetto a quello del 2011.

Tab.3: Dati su alcuni aggregati monetari in miliardi di lekë

\begin{tabular}{|c|c|c|c|c|c|c|}
\hline Anni & PIL, mld & Denaro fuori banca & M1 & M2 & M3 & BM \\
\hline 2007 & 967,670 & 154,956 & 242,675 & 506,137 & 761,001 & 228,230 \\
\hline 2008 & $1,089,293.00$ & 195,817 & 282,862 & 555,000 & $815,700.0$ & 271,729 \\
\hline 2009 & $1,148,082.00$ & 209,043 & 284,505 & 578,181 & $871,500.0$ & 284,839 \\
\hline 2010 & $1,222,462.00$ & 195,059 & 275,427 & 604,517 & $980,300.0$ & 283,453 \\
\hline 2011 & $1,282,254.00$ & 194,923 & 276,898 & 647,003 & $1,070,100.0$ & 289,809 \\
\hline 2012 & $1,307,899.00^{1}$ & 192,705 & 281,246 & 669,577 & $1,123,400.0$ & 297,722 \\
\hline Correl.a & PIL & -0.50 & -0.55 & 0.98 & 1.00 & 0.92 \\
\hline
\end{tabular}

Fonte: Banca dell'Albania. Rapporto statistico mensile 02/2013¹. 
II denaro fuori dalla banca è diminuito nel periodo 2010-2011 rispetto al 2008. La stessa situazione è accaduto con l'aggregato M1. Nel 2008 M1 è stato maggiore che nel 2010 ed il 2011. La base monetaria è aumentata. Questa crescita è dovuta soprattutto dall'aumento delle riserve di liquidità che dal denaro fuori banca. L'aggregato M3 è cresciuto. La ragione è l'aumento significativo dei depositi in valuta estera, circa il 50\% dei depositi totali. Questi cambiamenti si riflettono in percentuale nella tabella seguente:

Tab. 4: La dinamica in percentulae di alcuni indici monetari e del PIL

\begin{tabular}{|c|c|c|c|c|c|c|}
\hline \multicolumn{7}{|c|}{ Variazioni in percentuale } \\
\hline Anni & PIL, mld & Denaro fuori banca & M1 & M2 & M3 & BM \\
\hline 2008 & 112.6 & 126.4 & 116.6 & 109.7 & 107.2 & 119.1 \\
\hline 2009 & 105.4 & 106.8 & 100.6 & 104.2 & 106.8 & 104.8 \\
\hline 2010 & 106.5 & 93.3 & 96.8 & 104.6 & 112.5 & 99.5 \\
\hline 2011 & 104.9 & 99.9 & 100.5 & 107.0 & 109.2 & 102.2 \\
\hline 2012 & 102.0 & 98.9 & 101.6 & 103.5 & 105.0 & 102.7 \\
\hline
\end{tabular}

Dai dati di cui sopra vediamo due tendenze.

In primo luogo, gli aggregati monetari che si basano sul lek tassi di variano meno rispetto ai variazioni del PIL.

In secondo luogo, la variazione di M3, il quale comprende anche la valuta estera, è maggiore rispetto alla variazione del PIL.

Dai dati di cui sopra, si ricava anche la velocità di circolazione della moneta la quale non varia molto.

Tab. 5: La velocità di circolazione della moneta

\begin{tabular}{|c|c|c|c|c|}
\hline Anni & PIL/ Dfb & PIL/M1 & PIL/BM & PIL/M3 \\
\hline 2007 & 6.2 & 4.0 & 4.2 & 1.27 \\
\hline 2008 & 5.6 & 3.9 & 4.0 & 1.34 \\
\hline 2009 & 5.5 & 4.0 & 4.0 & 1.32 \\
\hline 2010 & 6.3 & 4.4 & 4.3 & 1.25 \\
\hline 2011 & 6.6 & 4.6 & 4.4 & 1.20 \\
\hline
\end{tabular}

In generale, la velocità di circolazione della moneta non cambia. Non ci sono motivi di cambiarla perché non è cambiato il modo dei pagamenti, o il periodo di pagamento dei dipendenti. Ė rimasto come era, un pagamento mensile. Non abbiamo un aumento delle carte di credito il che può influenzare sulla distribuzione del denaro nel tempo.

Cosa notiamo se mettiamo a confronto la variazione in aumento degli aggregati monetari con la crescita del PIL? Nel 2008 la variazione in aumento degli aggregati monetari è maggiore rispetto alla crescita del PIL. La quantità di denaro per 1 lekë di prodotto è superiore a tutti gli altri anni. In particolare per il periodo 2007-2012 abbiamo questa situazione.

Tab. 6: La quantità di denaro per 1 lekë del PIL nominale

\begin{tabular}{|c|c|c|c|c|c|c|c|}
\hline Anni & Dfb/PIL & M1/PIL & M2/PIL & M3/PIL & BM/PIL & Media & M3-M2 \\
\hline 2007 & 0.160 & 0.251 & 0.523 & 0.786 & 0.236 & 0.39 & 0.263 \\
\hline 2008 & $\mathbf{0 . 1 8 0}$ & $\mathbf{0 . 2 6 0}$ & 0.510 & $\mathbf{0 . 7 4 9}$ & $\mathbf{0 . 2 4 9}$ & 0.39 & $\mathbf{0 . 2 3 9}$ \\
\hline 2009 & 0.182 & 0.248 & 0.504 & $\mathbf{0 . 7 5 9}$ & $\mathbf{0 . 2 4 8}$ & 0.39 & $\mathbf{0 . 2 5 5}$ \\
\hline 2010 & 0.160 & 0.225 & 0.495 & $\mathbf{0 . 8 0 2}$ & $\mathbf{0 . 2 3 2}$ & 0.38 & $\mathbf{0 . 3 0 7}$ \\
\hline 2011 & 0.152 & 0.216 & 0.505 & $\mathbf{0 . 8 3 5}$ & $\mathbf{0 . 2 2 6}$ & 0.39 & $\mathbf{0 . 3 3 0}$ \\
\hline 2012 & 0.147 & 0.215 & 0.512 & $\mathbf{0 . 8 5 9}$ & $\mathbf{0 . 2 2 8}$ & 0.39 & $\mathbf{0 . 3 4 7}$ \\
\hline Correl. al PIL & $-\mathbf{- 0 . 9 6}$ & $\mathbf{- 0 . 9 9}$ & $\mathbf{0 . 0 3}$ & $\mathbf{0 . 9 8}$ & $\mathbf{- 0 . 9 6}$ & & $\mathbf{0 . 9 9}$ \\
\hline
\end{tabular}

Osservando attentamente la tabella si vedono alcune tendenze:

In primo luogo, i rapporti DFB (denaro al di fuori della banca) / PIL, $\mathrm{M}_{1} / \mathrm{PIL}$ e BM / PIL hanno una forte relazione negativa al PIL (i coefficienti di correlazione sono rispettivamente di -0.96 , di -0.99 e di -0.96 ). II rapporto M2/PIL ha un effetto molto debole 0 assente del tutto (il coefficiente di correlazione è di 0.03 ). Ci sono dei variazioni nel senso opposto, diminuiscono gli aggregati monetari e aumenta il PIL.

In secondo luogo, il rapporto M3/PIL e $\mathrm{M}_{3}-\mathrm{M}_{2}$ hanno una forte correlazione positiva rispetto al PIL (i coefficienti di 
correlazione sono di 0.98 e di 0.99). Questo significa che quando aumenta $\mathrm{M}_{3}$ e la valuta estera, aumenta il PIL. In terzo luogo, tutti gli indicatori che si basano sul lek sono in declino oppure sono stabili.

In quarto luogo, l'aggregato $\mathrm{M}_{3}$, il quale comprende la valuta estera, è in aumento.

Se costruiamo le curve delle relazioni $\mathrm{MB} / \mathrm{PIL}$ e $\mathrm{M}_{3}-\mathrm{M}_{2} / \mathrm{PIL}$, esse tenderanno a essere contrarie e quasi completamente inelastiche. Questo significa che gli aggregati monetari non sono stati influenti sulla crescita economica. L'impatto negativo degli aggregati monetari in lekë viene ammortizzato dal effetto positivo dell'aumento della valuta estera.

Fig. 4: La curva della relazione MB / PIL

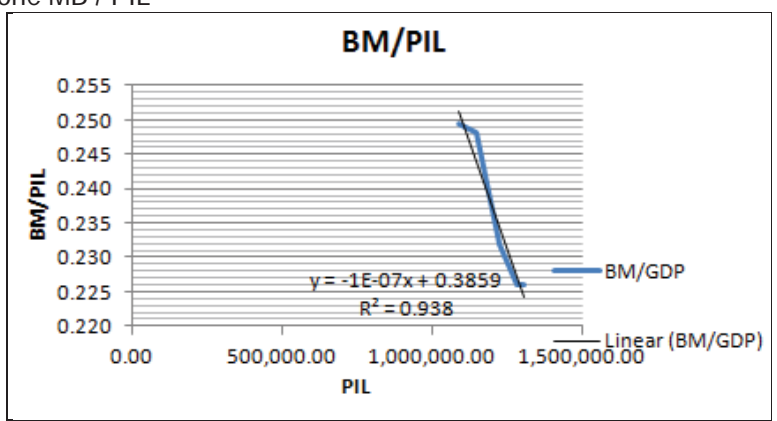

Fig. 5: La curva della relazioneM3-M2/PIL

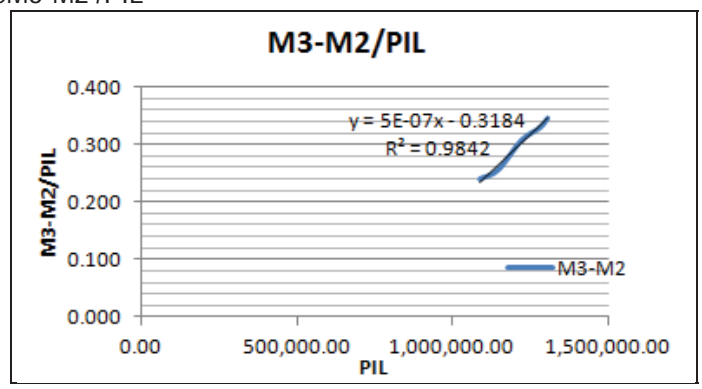

Le relazione di cui sopra dimostrano che una grande quantità di denaro segue la stessa quantità di beni nel 2008. Nell'anno 2008 c'è la maggiore crescita economica degli ultimi 8 anni. La conclusione è abbastanza semplice. L'aumento della quantità di denaro per una unità di beni comporta la crescita economica oppure la crescita economica viene accompagnata da un aumento della quantità di moneta in circolazione per unità di merce. Semplicemente, l'aumento della domanda aggregata promuove l'aumento del prodotto complessivo. Utilizzando gli aggregati monetari che hanno la moneta locale alla base, le conclusioni vengono presentati nel grafico seguente.

Fig. 6: La dinamica della quantità di denaro per un unità di beni

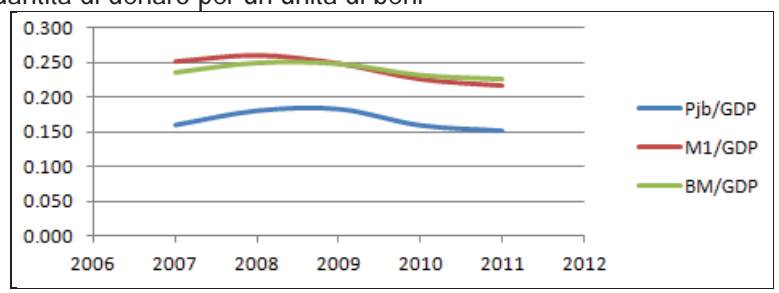

La posizione delle curve di relazione tra gli aggregati monetari ed il PIL è un altro indicatore che soddisfa i suddetti indicatori. 
Tab. 7: La variazione in percentuale negli anni

\begin{tabular}{|c|c|c|c|c|}
\hline & PIL & Dfb & M1 & BM \\
\hline 2009 & -7.2 & -19.6 & -16.0 & -14.2 \\
\hline 2010 & 1.1 & -13.4 & -3.8 & -5.3 \\
\hline 2011 & -1.6 & 6.6 & 3.7 & 2.7 \\
\hline 2012 & -2.9 & -1.1 & 1.0 & -3.5 \\
\hline
\end{tabular}

Dall'analisi di cui sopra, è chiaro che l'offerta di moneta è in diminuzione nella percentuale della sua crescita, per unità di merci, e generalmente in somma in assoluto.

La conclusione:

Se consideriamo l'aggregato M1 e la velocità del suo fatturato nel 2007, il quale è 4, allora l'economia albanese per gli anni 2009-2010- 2011 e il 20112 non ha avuto una vera crescita economica. Non consideriamo l'aggregato M3 perché una parte di esso va al di fuori dell'economia albanese sotto forma di depositi o di acquisto di attività finanziarie. All'interno una parte sostanziale di esso è servito nell'acquisto di immobili e allo scoperto di conto corrente.

II tasso di interesse non è stato influente, oppure è stato di molto poca influenza negli aggregati che hanno di base il lek, visto che dalla riduzione del tasso di interesse dal 6,25\% al 3,75\% invece di aumentare la quantità di leke in circolazione, si è ridotto oppure è rimasto costante.

Gli unici cambiamenti ci sono sulla quantità della valuta estera, il tasso base di esse viene determinato dalla Banca Centrale Europea e dalla FED Americana.

Un impatto sul PIL hanno avuto solo i tassi di cambio.

La combinazione delle curve IS e LM. Facendo riferimento ai modelli di cui sopra ed alle condizioni specificate per l'economia albanese per le due curve si può scrivere:

Fig. 7: L'equilibrio nei mercati dei beni e le attività

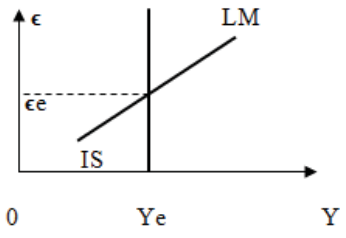

$Y=C+I(r)+G+N X(e) I S$

$M / P=L(r, Y) L=k y L M$

La prima equazione descrive l'equilibrio nei mercati dei beni e dei servizi. La seconda equazione descrive l'equilibrio nei mercati monetari.

Nei variabili endogeni includiamo il reddito $Y$ ed il tasso di cambio $\varepsilon$. Le due curve sono presenti nella Fig. 7. L'equilibrio macroeconomico per l'economia è stabilito nel punto in cui la curva IS incrocia la curva LM. Questa intersezione indica il livello del tasso di cambio e il reddito nei mercati in cui i beni e i servizi sono in equilibrio.

II grafico mostra come il reddito ed il tasso di cambio rispondono all'attuazione delle politiche economiche fiscali e monetarie. Esattamente il problema che ci opponiamo alla nostra analisi. Prenderemo in esame l'effetto delle politiche monetarie e fiscali in condizioni di tassi fissi e variabili.

\section{II Ruolo del Tasso di Cambio Flessibile sulla Crescita Economica in Una Piccola Economia Aperta}

L'economia Albanese è una piccola economia aperta. Si tratta di una economia in via di sviluppo ed in crescita, che è indietro in confronto agli altri paesi dell'Europa occidentale in industria, nell'agricoltura, nei servizi, nei trasporti, nel turismo, ecc. Si tratta di un paese consumatore, importatore dei prodotti di base, con un rapporto esportazioniimportazioni 1 / 2.3 (nel 2012). In Albania non esistono i mercati consolidati ed il mercato secondario dei titoli. Manca il capitale finanziario dalla fusione del capitale del settore industriale con quello bancario. Mancano i fenomeni economici che caraterizzano i paesi sviluppati, quindi non si possono manifestare gli effetti di trasmisione banca-imprese -borsa. Essendo un paese arretrato non ci sono quelle forze produttive e le relazioni economiche che esistono in Occidente, pertanto, non si può prendere come modello ogni cosa dall'ovest e applicarla in queste condizioni, come è accaduto in molte aree. La Banca Centrale di Albania ha preso come modello il suo principale obiettivo dalla Banca Centrale Europea 
che è "la preservazione della stabilità dei prezzi". Tale obiettivo in un paese come l'Albania non può essere attuato con successo, perché è un obiettivo normale per i paesi sviluppati che non hanno problemi con la crescita economica e la disoccupazione, che operano su un prodotto circa potenziale. L'economia albanese dovrebbe avere un obiettivo per la protezione del tasso di cambio della moneta locale con l'obiettivo della crescita economica.

Qui di seguito potrete vedere come le politiche fiscali e monetarie influenzano un paese come l'Albania il quale applica tassi di cambio flessibili.

La politica fiscale. Se il governo Albanese attua una politica fiscale espansiva, abbassando le tasse 0 aumentando la spesa pubblica, che cosa accadrebbe per l'economia? Porterà questa politica ad un aumento dei redditi, alla crescita economica, e nel miglioramento degli indicatori macroeconomici? Se l'economia Albanese fosse un'economia chiusa, una tale politica potrebbe aumentare le entrate. In una piccola economia aperta, il tasso di cambio flessibile, l'espansione fiscale mobile lascia allo stesso livello il reddito. Perché non sono aumentati i redditi nel caso dell'economia Albanese? La risposta è data dalla posizione delle curve. L'espansione fiscale lo vediamo in due casi.

In primo luogo, quando il governo agisce con il lek albanese sui tagli fiscali o sull'aumento della spesa pubblica. In questo caso non cambia la quantità di denaro in circolazione, e quindi non avremmo alcun impatto sulla variazione degli indicatori sia sul reddito che sull'inflazione. Possiamo vedere temporaneo aumento della domanda aggregata, ma senza alcun impatto sul livello dei redditi.

In secondo luogo, se il governo prende in prestito in valuta estera da soggetti economici esteri attraverso la vendita dei titoli o tramite prestiti allora avremmo reazioni temporanee sul mercato monetario.

La politica di espansione aumenterà la spesa pubblica. La spesa pubblica aumenterà temporaneamente la domanda aggregata e la crescita della domanda aggregata sposterà la curva IS a destra in alto come si vede nella fig.8. Il tasso di cambio del euro sarà deprezzato ed il livello dei reddito aumenterebbe temporaneamente dalla conversione della valuta estera con il lek. Ma l'esportazione della valuta all'estero e la riduzione del tasso di cambio porterebbe alla posizione precedente. Per questo l'aumento tende ad eleminarsi passando dal punto $\mathbf{c}$ al punto a nel punto $\mathbf{c}$ e termina al punto di partenza del reddito $\mathrm{IS}_{1}$.

In un'economia chiusaquando aumentano i ricavi, aumentano i tassi di interesse, perché la crescita del reddito fa crescere la domanda di moneta. Questo non è possibile in una piccola economia aperta, perché i tassi di interesse sarebbero immediatamente al di sopra del tasso di interesse del mondo $\mathrm{r}$, ed il capitale affluirà dall'estero. Nel caso dell'Albania non succede proprio cosi, perché il tasso di interesse del lek è sempre stato superiore al tasso di interesse dell'euro e del dollaro. Il tasso di interesse del lek ha mai tentato di seguire loro. In questo modo normalmente si dovrebbe avere un aumento della domanda di moneta. Allora cosa è successo? II tasso di tasso di interesse di base è sceso molto lentamente, dal 6,25\% che era nel 2009 al 3,75\% nel 2013. Questa diminuzione non è stata associata da una crescita degli aggregati monetari, al contrario, questo fenomeno, come abbiamo indicato sopra è avvenuto solo per $\mathrm{M}_{2}$ e $\mathrm{M}_{3}$ e gli altri aggregati o c'è stato il declino o sitazione è rimasta stagnante. In questo modo, nel nostro caso, anche se supponiamo l'economia Albanese come un'economia chiusa non c'è stata la manifestazione attesa. L'entrata della moneta estera in Albania non è avvenuto perché il tasso di interesse, ma per altri motivi. In particolare la moneta veniva ottenuta dalle esportazioni, dai emigranti che lavorano all'estero, dagli investimenti esteri, dalle vendite di beni dello Stato, dall'indebitamento del governo, ecc. Quindi, se si parla di flusso in entrata ed in uscita della moneta estera questi dipendono dai tassi di interesse dell'euro e del dollaro che circolano liberamente in Albania nello stesso modo del lek albanese. Sul conto dell'interesse offerto non c'è nessun apprezzamento, ma tutti gli stranieri offrono le monete estere che sempre più stanno lasciando l'Albania, sotto forma di depositi nelle banche all'estero o sotto forma di acquisto di attività finanziarie estere.

Fig.8: Un espansione fiscale ad un tasso di cambio flessi variabile

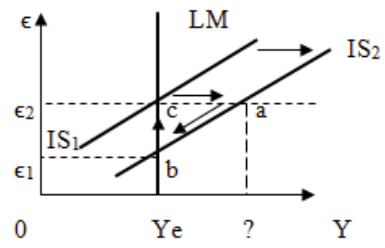

La valutazione del tasso di cambio farà in modo che i beni nazionali siano relativamente più costosi dei prodotti esteri, e ciò porterà ad una riduzione delle esportazioni nette. La riduzione delle esportazioni nette farà pressione sull'effetto della politica fiscale espansiva sul reddito. 
Perché il calo delle esportazioni nette è un fattore importante per rendere completamente ininfluente la politica fiscale sul reddito? In entrambi i casi sia nelle economie chiuse che quelle aprerte, la domanda reale e l'offerta di denaro si bilanciano.

Nell'economia albanese il tasso di interesse interno i è stato insensibile alla variazione dell' i* (il tasso di interesse mondiale), quindi è solo a un livello di reddito che può soddisfare questa equazione. Questo livello di reddito non può essere modificato quando cambia la politica fiscale. Così quando il governo aumenta la spesa oppure attua riduzioni fiscali, come è accaduto in Albania, l'apprezzamento del tasso di cambio del lek ed il calo delle esportazioni nette sono esattamente nella stessa dimensione sufficiente a influire completamente sull'effetto normale della politica espansiva dei redditi.

Tab.8: Gli indicatori di bilancio del governo

Le statistiche di bilancio del governo
\begin{tabular}{|l|l|l|l|}
\hline Anni & Ricavi & Costi & Saldo(-) \\
\hline 2007 & 251,555 & 285,674 & 34,119 \\
\hline 2008 & 291,238 & 351,492 & 60,254 \\
\hline 2009 & 299,597 & 379,602 & 80,005 \\
\hline 2010 & 324,721 & 362,752 & 38,031 \\
\hline 2011 & 330,475 & 376,352 & 45,877 \\
\hline 2012 & 330,383 & 376,239 & 45,856 \\
\hline
\end{tabular}

Fonte: Banca dell'Albania. Rapporto Statistico Mensile, 10/2011, 12/ 2012. P.5. Rapporto Statistico Mensile, 02/2013.

Per spiegare più chiaramente. L'aumento della spesa pubblica normalmente sposterà la curva IS a destra. Questo spostamento crea, attraverso l'aumento della quantità di moneta in circolazione, la svalutazione della valuta nazionale, il lek. Tale svalutazione provocherebbe un aumento delle esportazioni nette e, quindi, la crescita dei redditi. In realtà non è successo cosi nell'economia Albanese. Perché? La risposta è semplice. Perché la politica espansiva del governo che è stata presentata nella tabella n.8 non ha influenzato sulla crescita della quantità di moneta in circolazione, visto che non sono aumentati gli aggregati monetari. Ciò significa che il denaro è passato dalle imprese al governo in varie forme senza aumentare la quantità di moneta in circolazione. Così il non aumentarsi della quantità di denaro in circolazione porta alla non variazione dei tassi di cambio. Maggior impatto sulla variazione dei tassi di cambio ha la quantità di moneta estera in circolazione che la moneta lokale. Questo è stato dimostrato negli ultimi anni.

Se analizziamo i tassi di cambio per mesi per il periodo 2008-2012 si vedrà il che la moneta locale, il lek, viene stimato in Albania, nei periodi in cui gli immigrati vengono per le vacanze, di Natale, di capodanno 0 in estate. I dati sui tassi di cambio, per mesi, sono riportati in Tabella n. 9.

Tab. 9: I tassi di cambio dell'euro

\begin{tabular}{|c|c|c|c|c|}
\hline & 2008 & 2009 & 2010 & 2011 \\
\hline Gennaio & $\mathbf{1 2 2 . 6 1}$ & $\mathbf{1 2 5 . 1 8}$ & $\mathbf{1 3 8 . 2 8}$ & $\mathbf{1 3 8 . 6 5}$ \\
\hline Febbraio & 123.69 & 128.79 & 138.80 & 139.59 \\
\hline Marzo & 124.59 & 130.67 & 139.20 & 140.14 \\
\hline Aprile & 122.68 & 130.46 & 138.36 & 141.48 \\
\hline Maggio & 122.08 & 132.05 & 136.72 & 141.8 \\
\hline Giugno & 122.03 & 131.16 & 136.65 & 141.97 \\
\hline Luglio & $\mathbf{1 2 1 . 8 7}$ & $\mathbf{1 2 9 . 6 6}$ & 136.11 & 139.92 \\
\hline Agosto & $\mathbf{1 2 1 . 4 4}$ & 131.01 & 136.24 & 139.85 \\
\hline Settembre & 123.05 & 133.94 & 137.05 & 140.49 \\
\hline Ottobre & 123.13 & 136.90 & 138.39 & 140.81 \\
\hline Novembre & 123.29 & 137.70 & 138.82 & 140.97 \\
\hline Dicembre & 123.18 & 137.17 & 138.81 & 138.3 \\
\hline
\end{tabular}

Fonte: Banca dell'Albania. Rapporto Statistico, 10/2011, 12/ 2012. Rapporto Statistico Mensile, 02/2013.

Con i dati di cui sopra costruiamo i rispettivi grafici 
Fig. 9: Grafici dei tassi di cambio dell'euro

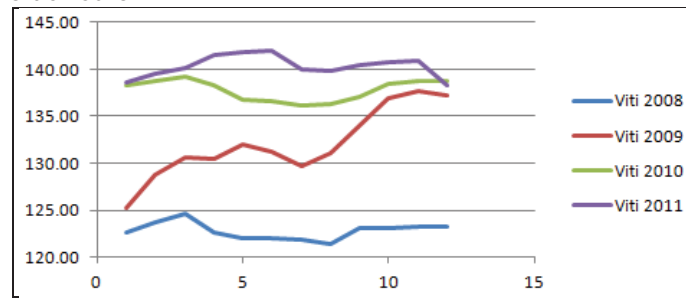

Dalla tabella e dai grafici si vede chiaramente che i tassi di cambio variano a seconda dell'arrivo degli immigrati. Nel periodo gennaio-luglio-agosto e dicembre, i tassi di cambio dell'euro sono bassi, perché questi sono i mesi che coincidono con l'arrivo degli immigrati. II contrario è accaduto con altri mesi in cui si rivaluta l'euro. Mentre per quanto riguarda il dollaro, ci sono dei cambiamenti, ma esso non ha molto peso nell'economia.

Allo stesso modo, siamo in grado di confrontare i tassi di scambio con la quantità di denaro fuori banca e la base monetaria.

Tab.10: Collegamento della BM e il Dfb con i tassi di cambio

\begin{tabular}{|c|c|c|}
\hline BM (media) & Dfb & Tasso di cambio EUR (medio) \\
\hline $\mathbf{2 5 9 , 7 6 5 . 8 0}$ & $182,324.4$ & 131.180 \\
\hline $\mathbf{2 5 9 , 0 3 4 . 4 0}$ & $182,648.2$ & 132.718 \\
\hline $\mathbf{2 5 7 , 6 8 6 . 7 6}$ & $182,131.8$ & 133.651 \\
\hline $\mathbf{2 5 7 , 8 6 9 . 3 2}$ & $182,494.6$ & 133.245 \\
\hline $\mathbf{2 5 8 , 3 1 4 . 5 9}$ & $182,135.3$ & 133.164 \\
\hline $\mathbf{2 6 1 , 2 4 9 . 8 4}$ & $184,129.7$ & 132.954 \\
\hline $\mathbf{2 6 4 , 3 8 2 . 1 2}$ & $186,987.8$ & 131.890 \\
\hline $\mathbf{2 6 4 , 9 2 7 . 5 6}$ & $186,665.5$ & 132.134 \\
\hline $\mathbf{2 6 5 , 8 7 0 . 8 1}$ & $183,850.2$ & 133.633 \\
\hline $\mathbf{2 6 8 , 4 8 5 . 4 2}$ & $185,864.2$ & 134.808 \\
\hline $\mathbf{2 6 9 , 9 1 1 . 5 1}$ & $187,658.0$ & 135.195 \\
\hline $\mathbf{2 8 2 , 4 5 7 . 5 5}$ & $198,710.6$ & 134.365 \\
\hline $\mathbf{0 . 5 1 9 8 1 5 2 8 6}$ & $\mathbf{0 . 3 7 8 7 9 2 9 5 4}$ & coeff. di correlazione \\
\hline
\end{tabular}

I dati mostrano che il rapporto tra loro è molto debole. Questo dimostra che i cambiamenti degli aggregati monetari basati sul lek non influiscono su i tassi di cambio. In questo modo le politiche della Banca Centrale nei confronti dei tassi di cambio non hanno alcun effetto sul risultato della crescita economica.

Fig.10: La dinamica della BM e ع per mesi nel periodo 2008-2011

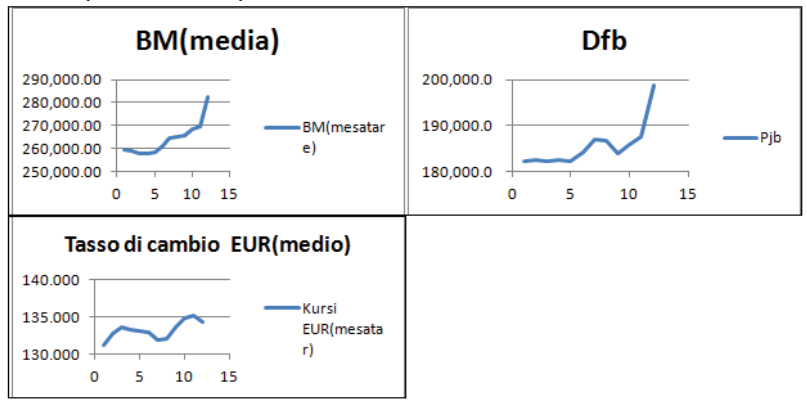

Anche dal diagramma sopra mostrata si vede chiaramente che il rapporto tra gli aggregati monetari basati sul lek e il tasso di cambi dell'euro è molto debole.

La conclusione. La politica espansiva del governo non è efficace nella crescita dei redditi se non è associata ad un aumento degli aggregati monetari. Essa realizza il passaggio del denaro da una parte ad altra aumentando 0 riducendo temporaneamente la domanda aggregata senza avere alcun impatto sui tassi di cambio. 
Quando il governo prende prestiti da soggetti economici esteri, aumentata la moneta estera in circolazione e viene apprezzato la moneta locale. La sua valutazione fa pressione sul reddito di passare nella posizione di origine senza modifiche.

La politica monetaria. Supponiamo ora che la Banca Centrale aumenta l'offerta di moneta. Quando si parla di aumento dell'offerta significa considerare gli aggregati monetari che dipendono dalla posizione della moneta locale e non dalla valuta estera come M3. Poiché il livello di prezzo l'abbiamo ipotizzato fisso, un aumento dell'offerta di moneta tramite la significativa riduzione del tasso di interesse (REPO), aumenterà i saldi reali. L'incremento delle partite reali sposterà la curva LM verso destra come si mostra nella Fig. 11. Aumentando così l'offerta di moneta aumenta il reddito ed il tasso di cambio. Quindi, sarà svalutata la moneta locale e aumenterano i redditi.

La politica monetaria influenza il reddito in un'economia aperta, invece in una economia chiusa non influisce, il meccanismo di trasmissione monetaria è diverso. Ricordiamo che in una economia chiusa un aumento dell'offerta di moneta fa aumentare i costi, perché riduce il tasso di interesse e stimola gli investimenti. Normalmente, in una piccola economia aperta, il tasso di interesse interno è fissato in relazione al tasso di interesse mondiale. Nel caso dell'economia albanese il tasso di base non ha seguito il ritmo dei cambiamenti dei tassi mondiali. Inoltre, anche i tassi di interesse dei depositi e dei prestiti in valuta nazionale non hanno seguito questo cambiamento del tasso di interesse di base, piuttosto riflettono le strategie delle banche commerciali per i periodi di medio termine. Loro hanno accompagnato le modifiche del tasso di cambio dell'euro in funzione delle sue variazioni dalla Banca Centrale Europea e da questi si sono determinati i tassi di interesse su depositi e prestiti. Lo stesso è accaduto al dollaro. Le banche hanno seguito la posizione del tasso di interesse che ha definito la Fed americana. Le variazioni dei tassi di interesse del lek non hanno avuto molto peso in questo, perché è certo il mercato di titoli del governo. Notiamo qui che la maggior parte dei depositi in lek va all'acquisto di titoli di Stato che per i prestiti. Oltre il $60 \%$ del prestito è in euro e in dollaro.

Fig. 11: L'espansione monetaria sul tasso di cambio variabile

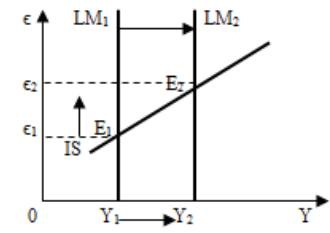

Un aumento dell'offerta di moneta sposta la curva LM a destra, abbassa il tasso di cambio e fa aumentare i redditi.

Questo effetto della crescita economica a seguito dell'aumento della quantità di moneta in circolazione, della crescita degli aggregati monetari non è stato molto evidente. La ragione è che questi aggregati non sono aumentati, e la Banca Centrale non ha avuto un impatto in questo settore. La diminuzione del tasso di cambio, il deprezzamento della valuta locale rende i beni nazionali relativamente più convenienti dei prodotti esteri e quindi stimola le esportazioni.

La conclusione: Nelle condizioni di una piccola economia aperta come l'economia albanese, la politica monetaria influenza i redditi dalla variazione del tasso di cambio, piuttosto che dalla variazione dei tassi di interesse.

\section{II Ruolo del Tasso di Cambio Fisso sulla Crescita Economica in Una Piccola Economia Aperta}

Nel precedente paragrafo abbiamo analizzato il ruolo delle politiche economiche monetarie e fiscali, in termini di un tasso di cambio variabile. In questa sezione si analizza il ruolo della politica economica nelle condizioni di un tasso di cambio fisso.

\subsection{II modello economico}

Se in Albania ci fossero i tassi di cambio fissi, la Banca Centrale acquisterebbe o venderebbe la moneta estera, l'euro 0 il dollaro, al fine di controllare il tasso di cambio. Supponiamo, per esempio, la Banca d'Albania annuncia che il tasso di cambio fisso è di 140 Lekë per 1 Euro (oppure 0,0071 Euro / 1 lekë). Questo significa che la Banca d'Albania è disposta a dare 140 lekë per 1 Euro o di dare 1 Euro in cambio di 140 lekë. In questo modo la Banca d'Albania è deve mantenere le riserve necessarie di moneta locare e estera, e che dovrebbe anche stampare per gli acquisti previsti.

II tasso di cambio fisso presuppone che la politica monetaria della Banca d'Albania ha un unico scopo, quello di mantenere il tasso di cambio ai livelli annunciati. In altre parole, l'essenza del sistema del tasso di cambio fisso è quello di permettere alla Banca d'Albania la regolazione dell'offerta di moneta in modo che il tasso di cambio di equilibrio sia 
pari al tasso di cambio pubblicato da essa tutti i giorni. In questo modo la Banca d'Albania è pronta ad acquistare 0 di vendere la moneta estera a un tasso di cambio fissato da essa. Utilizzando un tasso fisso l'offerta di moneta viene automaticamente regolata andando al livello necessario di mercato. Con un esempio concreto vedremmo come il tasso di cambio fisso determina l'offerta di moneta. Supponiamo che la Banca d'Albania annuncia il tasso di cambio 140 lekë per 1 Euro. Il tasso di cambio di mercato è 145 lekë per 1 Euro. Questa situazione è illustrata nella Fig. 11. Sottolineiamo che abbiamo un vantaggio opportunistico: nel mercato saranno acquistati 300 leke con 2 \$ e saranno venduti da parte della Fed per $3 \$$, con un profitto di $1 \$$. Quando la Fed compra questi leke al mercato, il dollaro pagato per loro aumenterà automaticamente l'offerta di moneta. L'aumento dell'offerta di moneta sposta la curva LM a destra, riducendo il tasso di cambio di equilibrio. In questo modo, l'offerta di moneta continuerà a crescere fino a quando il tasso di cambio di equilibrio diminuisca nel limite annunciato.

Fig.11: Come influisce il tasso di cambio fisso sull'offerta di moneta

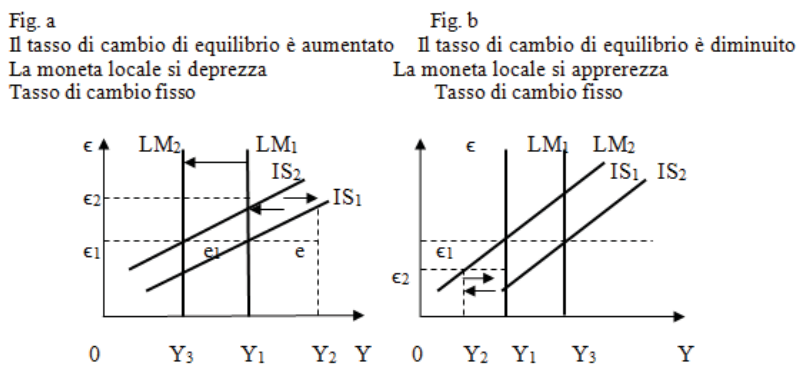

Nella figura a) il tasso di cambio di equilibrio supera inizialmente il livello fissato. Questo deprezzamento del lek inizialmente stimolerà le esportazioni e avremmo la crescita dei redditi. Per mantenere il tasso di cambio la Banca d'Albania venderà i titoli di stato e preleverà la moneta locale dalla circolazione. Riducendo l'offerta di moneta la curva LM passerà a sinistra a LM2 fino a quando il tasso di cambio del mercato sarà uguale al tasso di cambio della Banca. Diminuendo la quantità di moneta in circolazione comporterà la diminuizione dell'investimento in titoli di Stato e l'aumento in quelle delle banche. Quindi, se il governo ha portato ad aumentare l'offerta, la banca ha portato al suo declino, portando ad un tasso di cambio senza impatto sui redditi in equilibrio.

Nella figura b) ciabbiamo il caso inverso alla figura a), perché il tasso di cambio di equilibrio è al di sotto del livello fissato. Ciò significa che il lek è stato apprezzato e la moneta estera è stata deprezzata, che influenzerà temporaneamente sul calo dei redditi. La banca o i soggetti economici acquisteranno la moneta estera nei mercati di cambi della moneta. Sul mercato diminuirà la quantità di moneta estera e aumenterà la quantità di lek in circolazione. Così la curva LM1 si sposterà a destra sulla LM2. Questo processo aumenterà automaticamente il tasso di cambio della moneta estera raggiungendo il tasso annunciato dalla Banca d'Albania.

E 'importante capire che questo sistema di tassi di cambio fissi, fissa i tassi di cambio nominali. Se i prezzi sono variabili, quindi l'economia è in un periodo di lunga durata, in modo che il tasso di cambio reale può variare invece il tasso di cambio nominale rimane fisso. A lungo termine, la politica monetaria con tassi di cambio fissi non influirebbe in alcun indice reale, tra cui sul tasso di cambio reale. II tasso di cambio nominale fisso influenzerà solo l'offerta di moneta ed il livello dei prezzi. In un periodo di breve termine prescritto dal modello di Mundell-Fleming i prezzi sono fissi, ed il tasso di cambio nominale fisso comprende anche il tasso di cambio reale fisso.

\subsection{La politica fiscale}

In questa sezione esaminiamo specificamente gli effetti della politica fiscale in una piccola economia aperta con un tasso di cambio fisso. Si supponga che il governo albanese stimola la crescita della spesa interna attraverso i tagli fiscali 0 della crescita dei salari. Questa politica sposterà la curva ISO verso destra a IS ${ }_{1}$ (come mostrato nella fig.12), aumentando il tasso di cambio. L'aumento della quantità di lek in circolazione invalida essa, aumentando il tasso di cambio dell'euro. Inizialmente il reddito andrà a $Y_{2}$. La Banca Centrale è pronta a mantenere il tasso di cambio fisso in modo che risponda alla crescita del tasso di cambio, prelevando il lek attraverso la vendita di titoli alle entità economiche. Aumentando così I'offerta di moneta sposterà la curva LM a destra fino a $Y_{3}$, fino a stabilizzare il tasso di cambio. Quindi, l'espansione fiscale a tasso fisso aumenta il reddito aggregato, a differenza della situazione con il tasso di cambio variabile. 
La politica fiscale espansiva sposterà la curva $\mathrm{IS}_{1}$ a destra. Per mantenere il tasso di cambio fisso la Banca aumenterà I'offerta di moneta, quindi la curva $\mathrm{LM}_{1}$ si sposterà a destra. La conclusione è che la politica fiscale ha un effetto positivo sulla crescita economica in una piccola economia aperta con tassi di cambio fissi.

Fig.12: L'espansione fiscale a tassi di cambio fissi

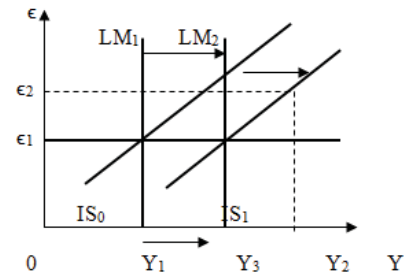

\subsection{La politica monetaria}

Si supponga che la Banca Centrale in condizioni di un tasso di cambio fisso aumenta l'offerta di moneta, dagli acquisti dei titoli dalle entità commerciali. Che cosa accadrà? L'impatto iniziale di questa politica sarà lo spostamento della curva LM a destra, aumentando i tassi di cambio come in Fig. 13. Dal momento che la Banca d'Albania deterrà il tasso di cambio invariato, rispondera rapidamente all'aumento del tasso di cambio, portando l'offerta di moneta e la curva LM nella posizione iniziale. Cosi la politica monetaria in termini di tassi di cambio fissi di solito non è efficace, perché nelle condizioni del tasso di cambio fisso la Banca Centrale ha il controllo sull'offerta di moneta.

Se la banca centrale aumenta l'offerta di moneta, per esempio, dall'acquisto dei Buoni del Tesoro dei soggetti economici, ciò consentirà l'aumento del tasso di cambio. Per mantenere fisso il tasso di cambio, l'offerta di moneta e la curva LM dovranno essere ritornare nella loro posizione di origine. Pertanto, la politica monetaria è inefficace sul tasso di cambio fisso.

Fig. 13: L'espansione monetaria con tassi di cambio fissi

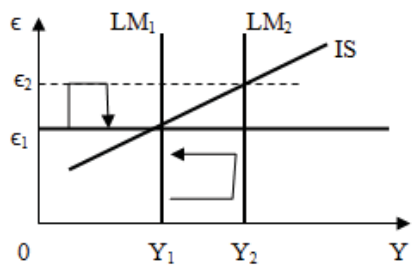

Sotto il regime di un tasso di cambio fisso, la politica monetaria avrà solo effetti sul reddito e sulla disoccupazione nazionale, non a lungo termine. Solo se accompagnata da una crescita della domanda aggregata che aumenta gli investimenti e diminuisce il risparmio, aumenterà l'offerta di moneta che porterà ad un aumento del livello del reddito nazionale.

\section{Conclusioni Generali}

In una piccola economia aperta, la curva delle esportazioni nette è inclinata positivamente. Questo perché la crescita delle esportazioni nette è stata determinata da un aumento delle esportazioni molto più veloce rispetto agli importazioni. La crescita delle esportazioni è avvenuto a seguito del deprezzamento della valuta locale.

La curva IS associata al tasso di cambio, è inclinata positivamente perché essa accompagna la curva delle esportazioni nette. Così, quando il Lek albanese si deprezza la curva dei costi si sposta in alto. Lo spostamentoin alto comporterà l'aumento dei redditi. Così, nell'economia albanese, esiste una correlazione positiva tra i tassi di cambio e i ricavi.

La curva LM è completamente verticale. Essa non dipende dai tassi di cambio. Se si considera il tasso di interesse degli ultimi 5-6 anni, si vede che non dipende dal tasso di interesse. La ragione è che in Albania operano sia l'euro che il dollaro nello stesso modo del lek.

II rapporto DFB (il denaro fuori banca) / PIL, M $1 /$ PIL e BM / PIL hanno una forte correlazione negativa al PIL. II rapporto $M_{3} / P I L$ e $M_{3}-M_{2}$ hanno una forte correlazione positiva al PIL. Questo significa che quando aumenta la valuta 
estera, aumenta il PIL.

Se consideriamo l'aggregato $\mathrm{M}_{1}$ e la velocità del suo fatturato nel 2007, il quale è 4, allora l'economia albanese per gli anni 2009-2010- 2011 e il 20112 non ha avuto una vera crescita economica. Non consideriamo l'aggregato $\mathrm{M}_{3}$ perché una parte di esso va al di fuori dell'economia albanese sotto forma di depositi o di acquisto di attività finanziarie. All'interno una parte sostanziale di esso è servito nell'acquisto di immobili e allo scoperto di conto corrente.

II tasso di interesse non è stato influente, oppure è stato di molto poca influenza negli aggregati che hanno di base il lek, visto che dalla riduzione del tasso di interesse dal 6,25\% al 3,75\% invece di aumentare la quantità di leke in circolazione, si è ridotto oppure è rimasto costante.

Gli unici cambiamenti ci sono sulla quantità della valuta estera, il tasso base di esse viene determinato dalla Banca Centrale Europea e dalla FED Americana.

La politica espansiva del governo non è efficace nella crescita dei redditi se non è associata ad un aumento degli aggregati monetari. Essa realizza il passaggio del denaro da una parte ad altra aumentando 0 riducendo temporaneamente la domanda aggregata senza avere alcun impatto sui tassi di cambio Quando il governo prende prestiti da soggetti economici esteri, aumentata la moneta estera in circolazione e viene apprezzato la moneta locale. La sua valutazione fa pressione sul reddito di passare nella posizione di origine senza modifiche.

Nelle condizioni di una piccola economia aperta come l'economia albanese, la politica monetaria influenza i redditi dalla variazione del tasso di cambio, piuttosto che dalla variazione dei tassi di interesse.

Il tasso di cambio nominale fisso influenzerà solo l'offerta di moneta ed il livello dei prezzi. variabile

L'espansione fiscale a tasso fisso aumenta il reddito aggregato, a differenza della situazione con il tasso di cambio

La politica monetaria, in termini di tassi di cambio fissi, di solito non è efficace, perché nelle condizioni del tasso di cambio fisso la Banca Centrale ha il controllo sull'offerta di moneta.

Pertanto, la politica monetaria è inefficace sul tasso di cambio fisso.

Sotto il regime di un tasso di cambio fisso, la politica monetaria avrà solo effetti sul reddito e sulla disoccupazione nazionale, non a lungo termine. Solo se accompagnata da una crescita della domanda aggregata che aumenta gli investimenti e diminuisce il risparmio, aumenterà l'offerta di moneta che porterà ad un aumento del livello del reddito nazionale.

\section{Bibliografia}

Prof.A. Angjeli, Prof.A.Kadriu, Doc.A.Naqellari. Makroekonomiks. Prima edizione, Editore: Università Internazionale di Struga, Macedonia, Università Mediterraneo di Tirana Albania. Macedonia 2010

N.Gregori Mankiw. Macroeconomia, quarta edizione, USA 2000

ABEL / Bernanke. Macroeconomia, terza edizione 1998. USA

Rudinger DORNBUCH \& Stanley FISCHER.1994. Edizione Internazionale. Tirana 2000. Macroeconomia

Rapporto statistico. Banca d'Albania. 03/2013. Tirana

Rapporto statistico. Banca d'Albania. 10/2012. Tirana

Annuario statistico. Ministero delle Politiche Agricole nel 2009

Annuario statistico dell'Albania 1991. Ministero dell'Economia. Dipartimento di Statistica.

II rapporto di politica monetaria per il terzo trimestre del 2012. Banca d'Albania

"La recessione globale ed il suo impatto sull'economia albanese". Monografia. Alqi Naqellari Robert Maliqi \& Almarin Naqellari, Tirana 2012.

Conferenza Internazionale di I. Alba - Scienze di Skopje 29-31 Agosto 2012. "Albanian economic model face pressure of global recession", A.Naqellari, T.Mehilli \& J. Shaqiri.

Conferenza Scientifica internazionale. "ALBANIA AND THE CRISIS: What's next? Revisiting the growth model" 15-16 dicembre 2012. "Gli effetti della politica monetaria sull'economia durante la recessione globale 2008-2011". Alqi Naqellari \& Michael Terida

L'Albania in cifre. 2010. INSTAT

Rapporto statistico mensile. 10/2011. Banca d'Albania 2011.

Documento di politica monetaria, 2012-2014. Banca d'Albania

Relazione Finanziaria di Stabilità per i primi 6 mesi del 2011. Banca d'Albania.

Risultati del sondaggio delle attività dei prestiti. Ottobre 2011. Banca d'Albania 